\title{
Models of Distribution of Electric Field of Primary Cilia as Monopole Antennas
}

\author{
Josef Dvorak ${ }^{1, *}$, Bohuslav Melichar ${ }^{2}$, Alzbeta Filipova ${ }^{3}$, Tomas Korinek ${ }^{4}$, Nela Grimova ${ }^{5}$, \\ Jana Grimova ${ }^{6}$, Aneta Rozsypalova ${ }^{1}$, Jan Proks ${ }^{1}$, Tomas Buchler ${ }^{1}$, Igor Richter ${ }^{7}$ \\ ${ }^{1}$ Department of Oncology, First Faculty of Medicine, Charles University and Thomayer Hospital, Prague, Czech Republic \\ ${ }^{2}$ Department of Oncology, Palacky University Medical School and Teaching Hospital, Olomouc, Czech Republic \\ ${ }^{3}$ Department of Radiobiology, Faculty of Military Health Sciences in Hradec Kralove, University of Defense in Brno, Hradec Kralove, Czech \\ Republic \\ ${ }^{4}$ Department of Electromagnetic Field, Faculty of Electrical Engineering, Czech Technical University, Prague, Czech Republic \\ ${ }^{5}$ Department of Cybernetics, Faculty of Electrical Engineering, Czech Technical University, Prague, Czech Republic \\ ${ }^{6}$ Medicon, Prague, Czech Republic \\ ${ }^{7}$ Department of Oncology, Regional Hospital, Liberec, Czech Republic
}

Email address:

josef.dvorak@ftn.cz (J. Dvorak)

${ }^{*}$ Corresponding author

\section{To cite this article:}

Dvorak Josef, Melichar Bohuslav, Filipova Alzbeta, Korinek Tomas, Grimova Nela, Grimova Jana, Rozsypalova Aneta, Proks Jan, Buchler Tomas, Richter Igor. Models of Distribution of Electric Field of Primary Cilia as Monopole Antennas. Journal of Cancer Treatment and Research. Vol. 6, No. 3, 2018, pp. 37-43. doi: 10.11648/j.jctr.20180603.11

Received: August 19, 2018; Accepted: October 11, 2018; Published: November 7, 2018

\begin{abstract}
Background: The primary cilium is a solitary, chemosensory and mechanosensory, non-motile microtubule-based organelle which in the quiescent cell cycle phase projects from the surface of most cells in vertebrates, including humans. A hypothesis has been proposed that the cell endogenous electromagnetic field results from a unique cooperating system among microtubules and mitochondria. The present study expands this prior hypothesis of the endogenous electromagnetic field in the cell to the present hypothesis that primary cilium could serve as a monopole antenna. It is proposed that primary cilia as monopole antennas can serve for both transmitting and receiving signals at the same frequency. Results: There was simulated the distribution of electric field of primary cilium as a monopole antenna of a single cell, primary cilia after mitosis and primary cilium of renal tubule in water environment. According to simulations of the distribution of electric field of primary cilium as a monopole antenna, the electromagnetic waves radiate not only to the neighbouring cells, but also to the nucleus of the cell proper where the gene expression during the cell cycle could be changed. Conclusions: The present study provides the first simulations of electromagnetic field of primary cilia as monopole antennas. The proof of this function of primary cilia could extend diagnostic and therapeutic modalities. There are several ways to verify this hypothesis. For example, it is possible to use the voltage sensitive dyes in the microenvironment outside the primary cilium or photon counting with low noise and highly sensitive photon counting system.
\end{abstract}

Keywords: Primary Cilia, Monopole Antennas, Distribution of Electric Field Models

\section{Introduction}

\subsection{Primary Cilia}

The primary cilium is a solitary, chemosensory and mechanosensory, non-motile microtubule-based organelle which in the quiescent cell cycle phase projects from the surface of most vertebrate (including human) cells, encompassing embryonal and stem cells (Figure 1). The centrosome migrates to the cell surface, in post-mitotic cells, where the mother centriole differentiates into the basal body. This structure nucleates microtubules forming the primary cilium. 


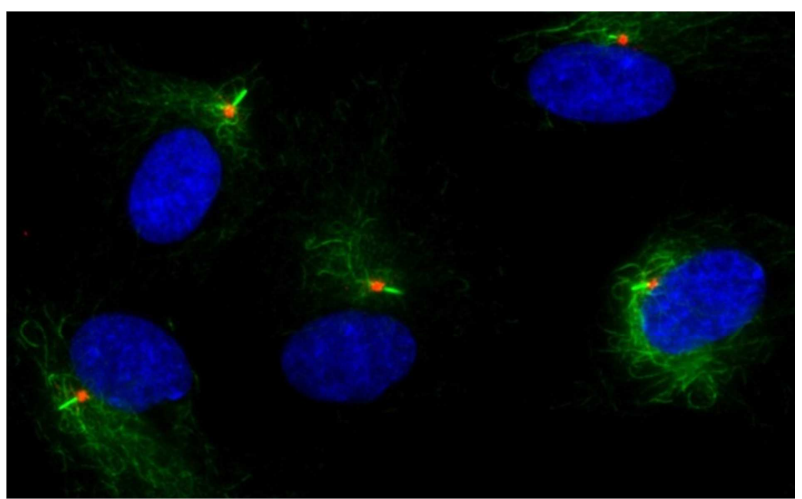

Figure 1. Primary cilia in vitro.

Human retinal pigment epithelial cells hTERT-RPE1 were cultivated $24 \mathrm{~h}$ in serum-free medium and thereafter fixed by formaldehyde, extracted by Triton X-100 and post-fixed by cold methanol. Primary cilia and cytoplasmic microtubules are stained by mouse monoclonal antibody to acetylated alpha-tubulin (green), centrosome by rabbit antibody to gammatubulin (red) and nucleus by DAPI (blue). Fluorescence microscope Olympus Provis AX70, objective 60x. Author: Dr. Eduarda Dráberová, Department of Biology of Cytoskeleton, Institute of Molecular Genetics, Czech Academy of Sciences.

Primary cilia respond to a variety of mechanical and molecular stimuli in the extracellular environment and transmit signals to the cell. Although the numbers and distribution depend on tissue type, primary cilia are detected in most tissues. Defects in the primary cilia structure and/or function have been shown to be causally linked to the development of a broad range of human diseases [1].

\subsection{Endogenous Electromagnetic Field in the Cell: Published Hypotheses About Mechanisms of Its Generation}

One of the most influential hypotheses explaining the mechanism of the generation of endogenous electromagnetic field (EMF) in the cell has been postulated by Herbert Fröhlich, who in 1968, proposed that biological systems display coherent longitudinal vibrations of electrically polar structures [2]. By definition electrically polar structures contain electrical charges and can, under certain conditions, generate EMF when vibrating [3]. This mechanism in Fröhlich's original model was general and not limited to a specific cellular structure.

One of the current modifications of Fröhlich's hypothesis is a unique cooperating system among mitochondria and microtubules, reviewed, for example, in reference [4]. Briefly, mitochondria are the power plants of the cell. Chemical energy stored in fatty acids and pyruvate is transformed into electrochemical proton gradient across the inner mitochondrial membrane, and ultimately stored into adenosine (ATP) and guanosine triphosphate (GTP). Protons transferred across the inner membrane diffuse through the outer membrane into the surrounding medium, and along with the negative charge in the mitochondrial matrix form a strong static electrical field around the mitochondria. The intensity of this static electric field at the outer mitochondrial membrane is about $3.5 \mathrm{MV} / \mathrm{m}$. In the vicinity of a single mitochondrion, the intensity of the electric field reduces almost linearly as a function of distance. Values of $540 \mathrm{kV} / \mathrm{m}$ for the electric field were measured at a distance of $2 \mu \mathrm{m}$ from mitochondrion $[5,6]$. About $40 \%$ of the input chemical energy of the mitochondrion are converted into ATP and GTP, with the remaining $60 \%$ liberated from mitochondria as photons and heat [4].

Microtubules are in a strong electric field since mitochondria are aligned near them. The microtubule is a highly dynamic polymer that continuously exchanges subunits (heterodimers) through the mechanism of dynamic instability (growing and shrinking of the microtubules) and by treadmilling (heterodimers are continually added at one end and lost from the other). One heterodimer binds 18 calcium ions. The electric dipole moment of heterodimers occurs at approximately $10^{-26} \mathrm{C} . \mathrm{m}$. The oscillations generate EMF. The strong electrostatic field surrounding the mitochondria can move microtubule oscillations into highly nonlinear region [5]. Mitochondrial dysfunction in cancer cells (Warburg effect) or in fibroblasts associated with cancer cells (reverse Warburg effect) results in decreased or increased power of the EMF generated, respectively [5, 7].

To the above-mentioned hypothesis concerning the endogenous EMF in the cell is added the present hypothesis of the possible function of primary cilium as a monopole antenna [8].

\section{A Hypothetical Model of Primary Cilium as a Monopole Antenna}

It is hypothesized, that the emitted and received wavelength of electromagnetic waves of primary cilium as a monopole antenna is proportional to the length of primary cilium, in the range between the length of the mother centriole/basal body in polarized centrosome after mitosis and the length of primary cilium during $\mathrm{G}_{0}$ phase. Centrioles/basal bodies are $0.25 \mu \mathrm{m}$ in diameter and their length may vary according to species and tissue type, around 0.4-0.5 $\mu \mathrm{m}$. The shortest wavelength could be the length of mother centriole/basal body in a polarized centrosome. The length of primary cilium during $\mathrm{G}_{0}$ phase is several microns, usually up to 10 microns, and only exceptionally longer. However, the emitted and received wavelength could be longer than the primary cilium. For example, in the technical applications the most common is the quarter-wave monopole antenna, in which the antenna is approximately $1 / 4$ of a wavelength of the electromagnetic waves [9].

Fundamental to the nature of antennas is that the electrical attributes remain consistent regardless whether they are receiving or transmitting, as stated by the reciprocity theorem of electromagnetics [9]. As a precondition for this reciprocity, the materials in the antenna and the transmission medium have to be both reciprocal and linear, meaning that the material responds consistently to an electric current or magnetic field from whichever direction. In all models proposed below is assumed this reciprocity. The reported 
values of conductivity of microtubules varies greatly in the literature [10], It is hypothesized, that primary cilia as monopole antennas can serve for both transmitting and receiving signals at same frequency (Figure 2).

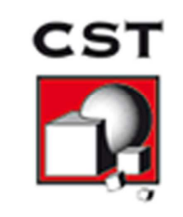

\begin{tabular}{|c|c|}
\hline \multicolumn{2}{|c|}{ e-field $(f=1.7)[1]($ peak $)$} \\
\hline Cutplane Name: & Cross Section A \\
\hline Cutplane Normal: & $1,0,0$ \\
\hline Cutplane Position: & 0 \\
\hline Component: & Abs \\
\hline 20 Maximum [V/m]: & $50.31 e+06$ \\
\hline Frequency: & 1.7 \\
\hline Phase: & 0 \\
\hline
\end{tabular}
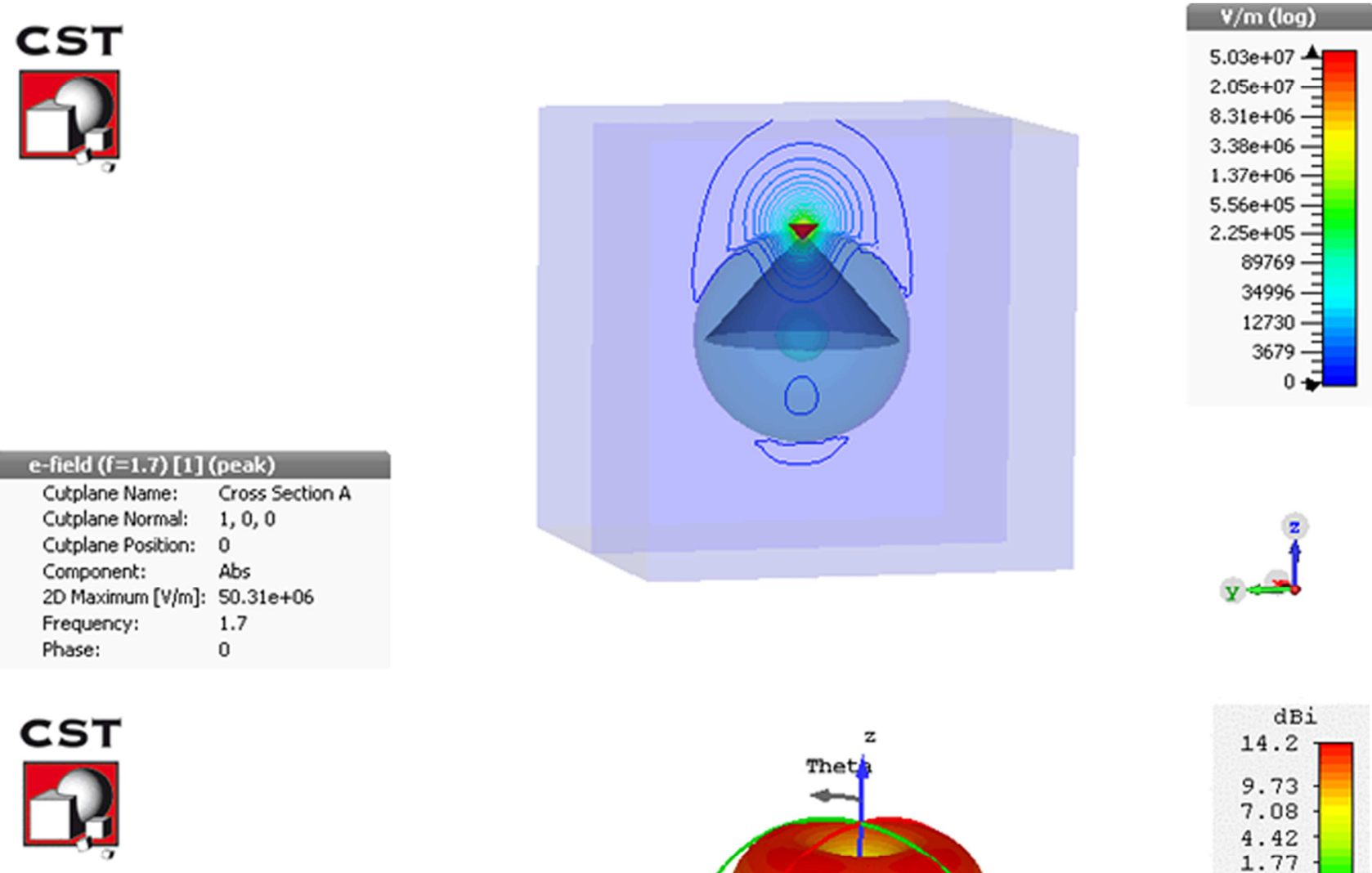

\begin{tabular}{|ll|}
\hline Type & Farfield \\
Approximation & enabled $(\mathrm{kR} \gg 1)$ \\
Monitor & farfield $(\mathrm{f}=1.7)[1]$ \\
Component & Abs \\
Output & Directivity \\
Frequency & 1.7 \\
Rad. effic. & $-36.26 \mathrm{~dB}$ \\
Tot. effic. & $-59.39 \mathrm{~dB}$ \\
Dir. & $14.15 \mathrm{dBi}$ \\
\hline
\end{tabular}
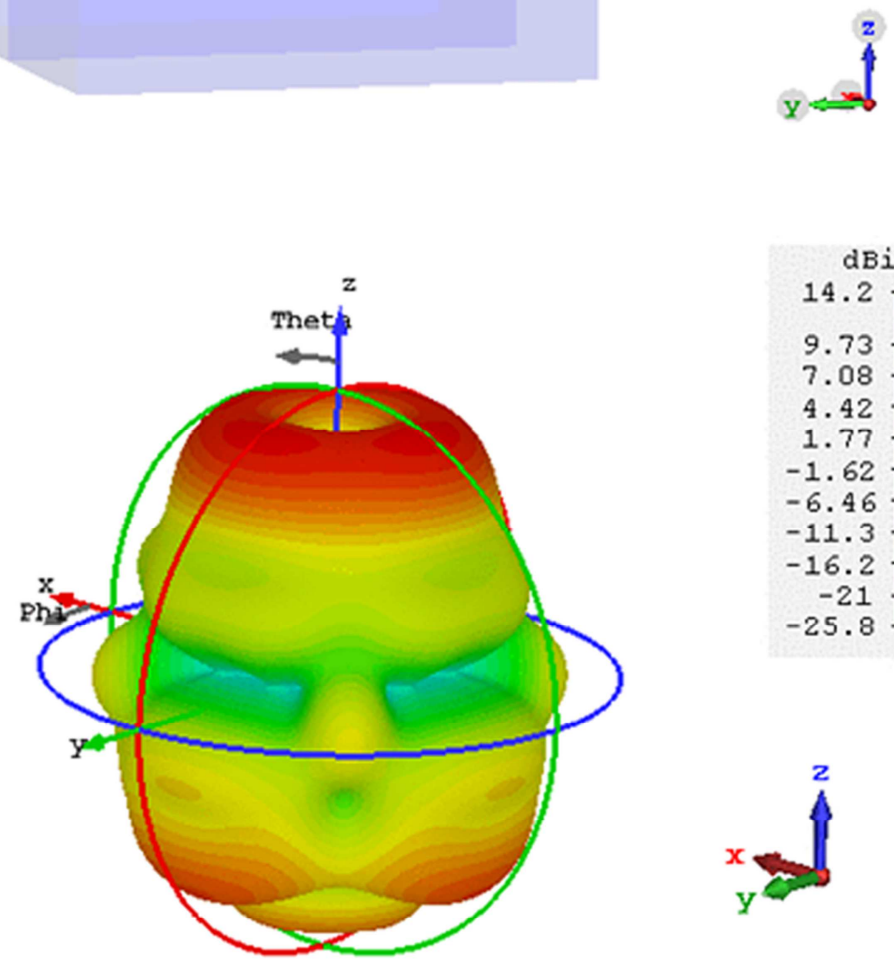

dBi

14.2

9.73

7.08

4. 42

1. 77

$-1.62$

$-6.46$

$-11.3$

$-16.2$

$-21$

$-25.8$

Figure 2. Primary cilium in silico.

a (top figure) Isoline plot of the distribution of electric field of primary cilium as a monopole antenna of a single cell.

b (bottom figure) Radiation pattern of endogenous EMF of primary cilium as a monopole antenna of a single cell.

The simulations was performed using software Computer Simulation Technology AG, Darmstadt, Germany (CST).

The overall power production of a living cell, determined with calorimetric measurements, is in the order of magnitude of $0.1 \mathrm{pW}\left(10^{-13} \mathrm{~W}\right)$ [11]. The radiation power of primary cilium as monopole antenna must be lower than this value. The radiated power is higher from a longer monopole antenna than from a shorter one [9], and it is hypothesized an analogous dependence in the primary cilium.

Ciliogenesis is an event regulated by the cell cycle. Primary cilia are found on the cells in $\mathrm{G}_{0}$ phase of the cell cycle. As the cells re-enter the cell cycle, the cilium is resorbed into the cytoplasm [12], and, consequently, this monopole antenna is formed and destroyed periodically. For example, the usual duration of cell cycle in cell cultures is about 24 hours. Mitosis takes less than one hour, and primary cilium as a monopole antenna in such cell culture can radiate electromagentic waves less than 23 hours during a period of 24 hours. The primary cilium is successively growing, therefore, the wavelength of this monopole antenna in each cell cycle period successively increases, and the frequency inversely decreases. In the beginning of the quiescent phase 
the primary cilium grows rapidly $(0.12 \mu \mathrm{m} / \mathrm{min}-0.4$ $\mu \mathrm{m} / \mathrm{min}$ ) [13], then in the plateau phase grows very slowly, but continuously, because the primary cilium can only grow, not decrease in length as it can be only irreversibly rapidly resorbed before mitosis. At this moment, a monopole antenna stops radiating. A resorption of primary cilium (accompanied with the shortening of the radiated wavelength) is faster than its formation. The exact moment of the resorption of primary cilium during the cell cycle depends on the cell type. Some cell types resorb primary cilium at the transition of $\mathrm{G}_{0} / \mathrm{G}_{1}$ phase, other cell types in S-phase or at the transition of $\mathrm{G}_{2} / \mathrm{M}$ phase [14]. In case of unfavorable conditions, such as starvation (e.g. after serum starvation in the cell culture), the length of primary cilium extends. It is hypothesized, that the changes of the length of the primary cilium and the velocity of these changes during the cell cycle are proportional to the wavelength and inversely to the frequency of emitted electromagnetic waves.

If the irradiated field is not electromagnetic, but is some manifestation of so-called new physics (e.g. warm dark matter), there would not be a valid radiation pattern of electomagnetic fíeld ( $f=c / \lambda$ ), but should be valid in the periodicity of cyclical presence of radiation of antenna structure of primary cilium during cell cycle and the dynamics of the changes its length.

After the mitosis there are two daughter cells, with two antennas. These antennas are not usually formed (cannot start to emit and receive signals) simultaneously, but the daughter cell with the older mother centriole usually forms primary cilium as a monopole antenna for several hours [15] earlier than the daughter cell with the mother centriole (Figure 3). Asymmetric inheritance of the centrosome which contains the mother centriole into one daughter cell is linked to maintaining stem cell character in the Drosophila germline, the developing mouse neocortex [16]. Primary cilia reassemble preferentially in vertebrate muscle specific stem cells, named satellite cells [15]. Primary cilia may function as positional sensors [17], and asynchronous cilium growth may bias the orientation of sister cells with regard to the stem cell niche [15].
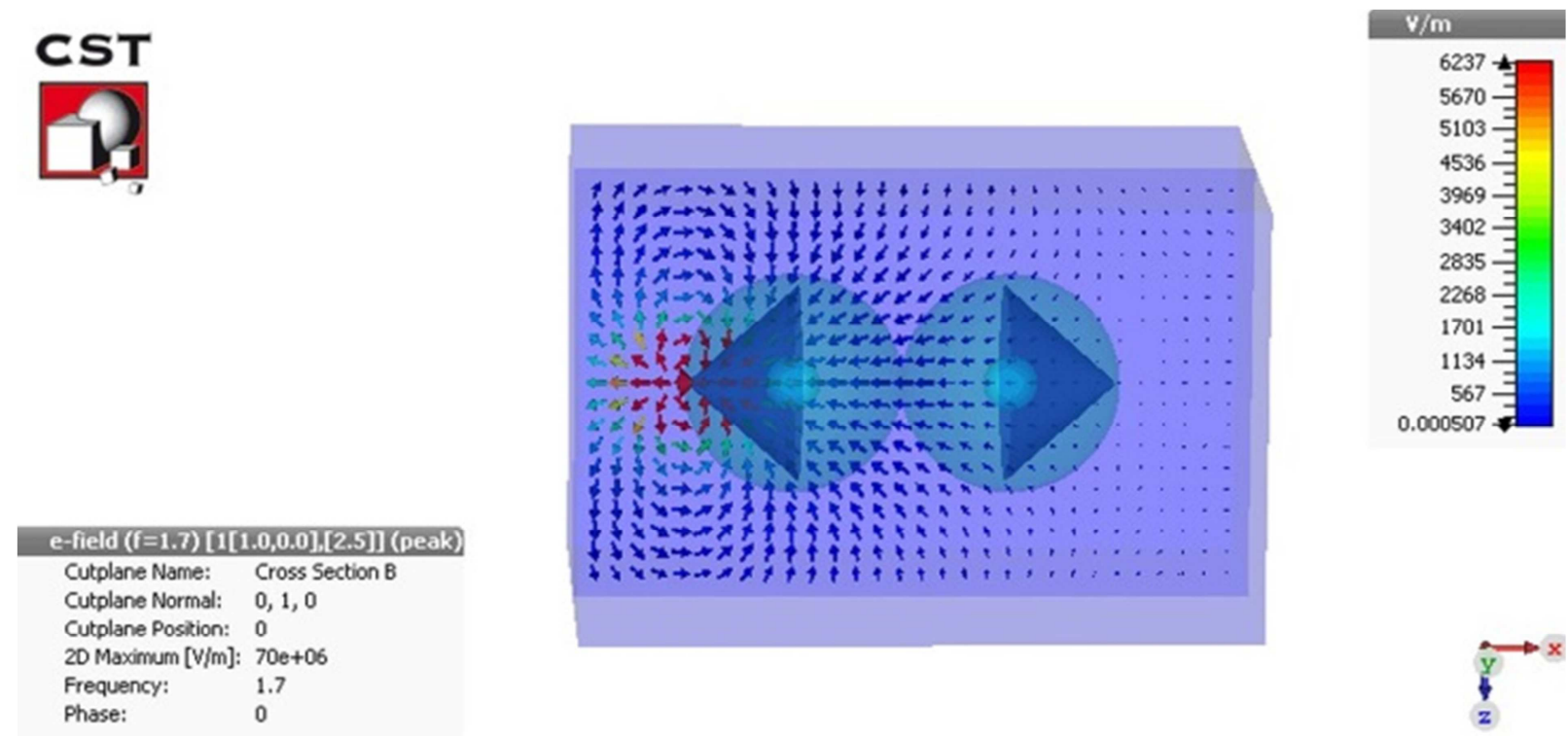

Figure 3. Primary cilium as a monopole antenna of the daughter cell.

Arrow plot of the distribution of electric field of primary cilium as a monopole antenna of the daughter cell with the older mother centriole shortly after mitosis. The simulation was performed using software Computer Simulation Technology AG, Darmstadt, Germany (CST).

It is hypothesized, that from the known location and other known parameters of primary cilia, it is possible to calculate the distribution of electric field and the radiation patterns of monopole antenna arrays in a water environment.

\section{Simulations of the Distribution of Electric Field and the Radiation Pattern of Electromagnetic Field of Primary Cilia as Monopole Antennas}

All simulations were performed using the software Computer Simulation Technology AG, Darmstadt, Germany (CST). All models are simulated in a water environment. For the simulation of the propagation of EMF in water the Debye model was used (Figure 2 - Figure 4). There has been simplified the structure of microtubules in the shape of a cone in the models.

\section{Discussion}

The present study provides the first simulations of EMF of primary cilia as monopole antennas. A majority of protein molecules are electrically polar structures. Even if the radiated power of the primary cilia is low, it may have an impact on intracellular processes such as the transport of substrates, temporal and spatial organization structures and molecules. It may provide an intracellular and extracellular 
signaling mechanism [18], may locally change the chemical reaction rates by attracting or rotating the molecular reaction partners [19].

The best transmission of signal by electromagnetic waves is between the same types of antennas, such as monopole antennas. The transmission of signal is possible also between two different types of antennas, for example, between monopole and dipole antenna, but the transmission signal is in principle not so good as between two same type antennas [20]. Therefore, it is hypothesized that the main target of the radiated signal of primary cilia as monopole antennas are other primary cilia.

According to simulations of the distribution of electric field of primary cilium as a monopole antenna, the electromagnetic waves radiate not only to the neighbouring cells, but also in the nucleus of the cell proper (Figure 2). In this context published experiments about the changes of gene expression caused by non-thermal effects of terahertz radiation are of interest [20]. In contrast to the published experiments, the emitted energy of primary cilium to the nucleus of the same cell is very small, but operates in close proximity of few microns over the long period of the

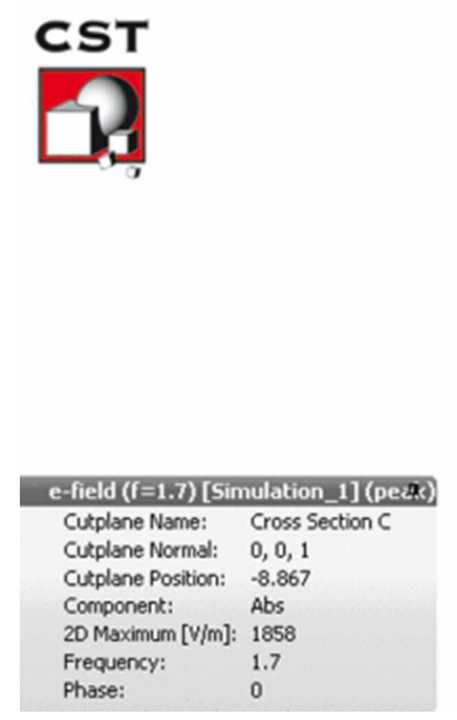

quiescent state. Therefore, it is hypothesized that it could be a changing the gene expression during the cell cycle of same cell.

As water interfaces with hydrophilic surfaces it undergoes structural change, which includes the many hydrophilic surfaces within the cell. This interfacial water has become known as "exclusion zone water" or "fourth-phase water". Cell water is mainly interfacial water [21]. In cell biology, it may play a role more fundamental than generally assumed [4]. It was shown, that formation of interfacial water is promoted by infrared radiation [21]. Therefore, it is assumed that infrared radiation of primary cilia could promote formation of interfacial water in the cells according to the distribution of electric field.

The majority of cancer cells lack the primary cilia, or possess these structures damaged or in a low frequency [22, 23]. Therefore, it would not be possible communicate with most cancer cell types, but only with primary cilia of cancer associated fibroblasts, which are present, in varying amount [24], in the tumor microenvironment of all solid tumors (Figure 4).

Figure 4. Primary cilia as monopole antennas of cancer associated fibroblasts.

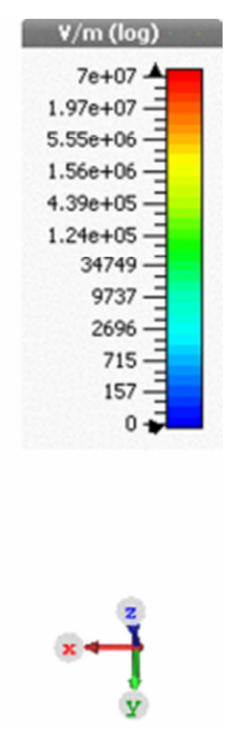

Isoline plot of the distribution of electric field of primary cilia as monopole antennas of cancer associated fibroblasts on the periphery and cancer cells without primary cilia in the middle of monolayer epithelium The simulation was performed using software Computer Simulation Technology AG, Darmstadt, Germany (CST).

There is an exception to this rule in the tumors dependent on the activating mutation of the Hedgehog signaling pathway. These tumors form the primary cilia at an increased frequency (e.g. small part of medulloblastoma and basalioma types) [25]. In these types of tumors it could be possible to communicate even with primary cilia of cancer cells.

\subsection{The Potential Implications of the Function of Primary Cilia as Monopole Antennas in the Light of This Hypothesis}

The proof of the function of primary cilia as monopole antennas could advance the understanding of the nature of life from molecular biology and chemistry deeper into the physics. It could extend diagnostic and therapeutic modalities. Disorders of the primary cilia are involved in the pathophysiology of a wide range of human diseases, from rare multisystem syndromes and developmental defects to the most common diseases such as cardiovascular disease, polycystic kidney disease, osteoarthrosis, and certain types of obesity $[14,26]$. Temporary primary cilia formation is essential for the successful repair of injury of certain cell types [27].

At present an important limitation of a deeper breakthrough between biotechnologies and microelectronics 
is the mutual interface. The communication between low frequency ionic currents via ion channels in biological tissues and high frequency currents in semiconductor materials in microelectronics is difficult. The communication would be much easier between two monopole antennas, one represented by primary cilium, and the second one artificial microelectronic, or even among monopole antenna arrays. Artificial monopole antennas of prosthetic devices could communicate with primary cilia as monopole antennas of healthy cells, including brain cells. In this context, it is notable, that almost all brain neurons possess primary cilia [28]. Neuronal primary cilium can transmit signal at a higher frequency than conduction velocity of an action potential, which is limited by the repolarization. Being an all-or-none action potential signal does not decay with transmission distance in contrast to signal from primary cilium which rapidly decays with transmission distance.

\subsection{Experimental Tests for Verification of the Function of Primary Cilia as Monopole Antennas}

There are several potential approaches to verify the present hypothesis. For example, it is possible to use the voltage sensitive dyes (nanosized voltmeter) in the microenvironment outside the primary cilium [6] or indirect cellular EMF detection by micro-dielectrophoresis [29]. During the measurement, the cells must be at room temperature, so that they are alive and able to communicate. The principal technical problem is a very weak radiation power of primary cilium (less than $10^{-13} \mathrm{~W}$ ) at the wavelength of several microns measured at background room temperature.

It is assumed that the measurement of the power of electromagnetic waves of microtubules of the cell could be easier through the primary cilia than inside the cell [30]. In the radial arrangement of microtubule dipoles, with negative electric charge on microtubule organizing center (MTOC), and positive electric charge in the periphery of the cell, with central location of the centrosome (MTOC) in the cell, the maximum power of EMF radiated is in the cell volume. However, in the case of polarization of the centrosome (MTOC) to the periphery of the cell, with microtubule axonema of primary cilium shaped as a monopole antenna, the radiation power of the EMF may be emitted into the surroundings of the cell.

From the various parameters of the EMF the power is the easiest to be measured. Because the radiation power of one primary cilium is extremely low, it would be easier to compare the power of EMF on a Petri dish between the monolayer cell culture of the same surface size (i.e. approximately the same number of cells) with and without the primary cilia. The volume of one cell can be microscopically measured and the number of cells on the Petri dish with the given surface can be approximated. The final length of primary cilia and the portion (percentage) of cells with primary cilia can be assessed immunofluorescently.

For the cell culture with the final length of primary cilia up to $0.9 \mu \mathrm{m}$ it is possible to use photon counting with low noise and highly sensitive photon counting system capable of spectral analyses. This system, located in a dark room, consists of a light-tight sample chamber, a photomultiplier tube, a rotating wheel with a set of glass filters for spectral analysis and computer-controlled photon counter. Electronic noise in a photomultiplier tube can be decreased by water cooler or thermoelectrically. It is expected a more sensitive experimental set-up with a photomultiplier tube above a Petri dish with cell culture (down view), than beside a Petri dish (side view).

For wavelengths longer than $0.9 \mu \mathrm{m}$ the dark current (electronic noise) of photomultiplier tubes is excessively high. Therefore it is necessary for the measurement of power in the wavelength of several microns to use a semiconductor near infrared detector matched to its preamplifier. It should be more sensitive to use a set of semiconductor detectors with various filters for different wavelengths according to the final length of primary cilia in the particular cell culture, e.g. semiconductor detectors sensitive in the range $0.9-2 \mu \mathrm{m}, 2-5$ $\mu \mathrm{m}$ and 5-7 $\mu \mathrm{m}$, respectively. Electronic noise in a semiconductor near infrared detectors can be decreased by liquid nitrogen in a Dewar flask or thermoelectrically. It is expected a more sensitive experimental set-up with a down view Dewar flask above the Petri dish with cell culture, than with a side view Dewar flask beside the Petri dish.

In the cell culture experiments is expected a higher photon emission in case of photon counting and higher radiation power of EMF in the case of semiconductor detectors in the wavelength proportional to the final length of primary cilia on the Petri dish with monolayer cell culture with primary cilia, compared to the same culture without primary cilia.

\section{Conclusions}

In conclusion, it is hypothesized that the primary cilia as monopole antennas can serve for transmitting and receiving signals and as positional sensors. The weak radiated power of EMF of primary cilia may also have an impact on intracellular processes in the same cell, proportionally to the changing length of primary cilium during the cell cycle. The present study provides the first simulations of the electromagnetic field of primary cilia as monopole antennas in a water environment. This hypothesis could be verified by voltage sensitive dyes (nanosized voltmeter) or photon counting.

\section{Acknowledgements}

Computer Simulation Technology AG, Branch Office Prague is acknowledged for the support. This study was supported by MH CZ-DRO (TH, 0064190) and Ministry of Defence of the Czech Republic - long-term organization development plan Medical Aspects of Weapons of Mass Destruction of the Faculty of Military Health Sciences, University of Defence. 


\section{Conflict of Interest Statement}

All the authors do not have any possible conflicts of interest.

\section{References}

[1] Basten SG, Giles RH. Functional aspects of primary cilia in signaling, cell cycle and tumorigenesis. Cilia 2013; 2:6.

[2] Fröhlich H. Long-range coherence and energy storage in biological systems. Int J Quant Chem 1968; 2:641-49.

[3] Cifra M, Fields JZ, Farhadi A. Electromagnetic cellular interactions. Prog Biophys Mol Biol 2011; 105:223-46.

[4] Pokorny J, Vedruccio C, Cifra M, Kucera O. Cancer physics: diagnostics based on damped cellular elastoelectrical vibrations in microtubules. Eur Biophys J 2011; 40:747-59.

[5] Pokorny J, Pokorny J, Foletti A, Kobilkova J, Vrba J, Vrba J. Mitochondrial dysfunction and disturbed coherence: gate to cancer. Pharmaceuticals (Basel) 2015; 8:675-95.

[6] Tyner KM, Kopelman R, Philbert MA. "Nano-sized voltmeter" enables cellular-wide electric field mapping. Biophysical Journal 2007; 93:1163-11.

[7] Pokorny J, Pokorny J, Borodavka F. Warburg effect-damping of electromagnetic oscillations. Electromagn Biol Med 2017; $36: 1-9$.

[8] Dvorak J, Melichar B, Filipova A, Grimova J, Grimova N, Rozsypalova A, et al. Simulations of centriole of polarized centrosome as a monopole antenna in immune and viral synapses. Special article J BUON 2018; 23(2):514-21.

[9] Balanis CA. Antenna Theory: Analysis and Design, 4th ed. Hoboken, New Jersey, USA: John Wiley \& Sons, Inc.; 2016.

[10] Minoura I, Muto E. Dielectric measurement of individual microtubules using the electroorientation method. Biophys $\mathrm{J}$ 2006; 90: 3739-48.

[11] Lamprecht I. Growth and metabolism in yeasts. In: Beezer AE, editor. Biological microcalorimetry. London: Academic Press; 1980. p. 43-112.

[12] Pan J, Snell W. The primary cilium: keeper of the key to cell division. Cell 2007; 129:1255-7.

[13] Lefebvre PA, Nordstrom SA, Moulder JE, Rosenbaum JL. Flagellar elongation and shortening in Chlamydomonas. IV. Effects of flagellar detachment, regeneration, and resorption on the induction of flagellar protein synthesis. J Cell Biol 1978; 78:8-27.

[14] Izawa I, Goto H, Kasahara K, Inagaki M. Current topics of functional links between primary cilia and cell cycle. Cilia $2015 ; 4: 12$.

[15] Anderson CT, Stearns T. Centriole age underlies asynchronous primary cilium growth in mammalian cells. Curr Biol 2009; 19:1498-502.
[16] Yamashita YM, Mahowald AP, Perlin JR, Fuller MT. Asymmetric inheritance of mother versus daughter centrosome in stem cell division. Science2007; 315:518-21.

[17] Benzing T, Walz G. Cilium-generated signaling: a cellular GPS? Curr Opin Nephrol Hypertens 2006; 15:245-9.

[18] Priel A, Tuszynski, Cantiello H. Electrodynamic signaling by the dendritic cytoskeleton: toward an intracellular information processing model. Electromagnetic Biology and Medicine 2005; 24: 221-31.

[19] Havelka D, Kucera O, Deriu MA, Cifra M. Electro-acoustic behavior of the mitotic spindle: a semi-classical coarsegrained model. PLoS One 2014; 9:e86501. doi: 10.1371/journal.pone.0086501.

[20] Alexandrov BS, Rasmussen KØ, Bishop AR, Usheva A, Alexandrov LB, Chong S, et al. Non-thermal effects of terahertz radiation on gene expression in mouse stem cells. Biomed Opt Express 2011; 2:2679-89.

[21] Chai BH, Zheng JM, Zhao Q, Pollack GH. Spectroscopic studies of solutes in aqueous solution. J Phys Chem A 2008; 112:2242-7.

[22] Menzl I, Lebeau L, Pande R, Hassounah NB, Li FW, Nagle R, Weihs K, McDermott KM. Loss of primary cilia occurs early in breast cancer development. Cilia 2014; 3:7.

[23] Gradilone SA, Radtke BN, Bogert PS, Huang BQ, Gajdos GB, LaRusso N. HDAC6 inhibition restores ciliary expression and decreases tumor growth. Cancer Res 2013; 73:2259-70.

[24] Dvorak J, Hadzi Nikolov D, Dusek L, Filipova A, Richter I, Buka D, et al. Prognostic significance of the frequency of primary cilia in cells of small bowel and colorectal adenocarcinoma. J BUON 2016; 21:1233-41.

[25] Wong SY, Seol AD, So PL, Ermilov AN, Bichakjian CK, Epstein EH Jr, et al. Primary cilia can both mediate and suppress Hedgehog pathway-dependent tumorigenesis. Nat Med 2009; 15:1055-61.

[26] Lee H, Song J, Jung JH, Ko HW. Primary cilia in energy balance signaling and metabolic disorder. BMB Rep 2015; 48:647-54.

[27] Jain R, Pan J, Driscoll JA, Wisner JW, Huang T, Gunsten SP, et al. Temporal relationship between primary and motile ciliogenesis in airway epithelial cells. Am J Respir Cell Mol Biol 2010; 43:731-9.

[28] Fuchs JL, Schwark HD. Neuronal primary cilia: a review. Cell Biol Int 2004; 28:111- 8 .

[29] Pohl HA. Braden T, Robinson S, Piclardi J, Pohl DG. Life cycle alteration of the micro-dielectrophoretic effects of cells. J Biol Phys 1981; 9:133-54.

[30] Kucera O, Cifra M, Pokorny J. Technical aspects of measurement of cellular electromagnetic activity. Eur Biophys J 2010; 39:1465-70. 